\title{
Managing impotence in diabetes
}

\author{
Should be part of a diabetes care service
}

Erectile impotence affects over one third of diabetic men. ${ }^{1}$ Effective treatments have been available for over a decade, yet it remains the most neglected complication of diabetes. It is not discussed in the British Diabetic Association's current guidelines for diabetes care ${ }^{2}$ or in its recent proposals for the audit of diabetes services. ${ }^{3}$ Most impotent men want help but are reluctant to discuss the problem. ${ }^{1}$

Few people would dispute that impotence can cause great unhappiness-so why has the problem been neglected for so long? It is difficult to resist the conclusion that the explanation lies in the previous widespread reluctance to discuss sexual problems. With inhibitions about sexual matters gradually changing, should impotence now be regarded as another diabetic complication and be managed routinely as part of a diabetes care service?

Erectile failure in diabetes is progressive and usually irreversible. ${ }^{4}$ Autonomic neuropathy is probably the most important aetiological factor, but arterial insufficiency may also contribute. ${ }^{5}$ Psychological problems are common in impotent men-indeed, impotence may cause psychological dysfunction. But there is no evidence that psychological problems are commonly the main cause of impotence in diabetes. Psychosexual counselling alone rarely restores potency. ${ }^{6}$

Although impotence in diabetes cannot be cured, several effective treatments exist. Penile prostheses have been available for many years, and good results have been reported in selected patients. ${ }^{7}$ Provision of prostheses by the NHS, however, is unusual as they are expensive and require an operation under general anaesthesia. For most doctors treating impotence two methods of treatment are available: intracavernosal injection and vacuum tumescence.

Intracavernosal injection of a vasoactive agent such as papaverine is widely used. The patient should be taught how to inject himself and should be given a test dose in hospital (usually $15 \mathrm{mg}$ ). The dose is incrementally increased until a satisfactory erection is achieved. This may require several hospital visits, but well motivated patients can experiment with increasing doses at home. Papaverine is not licensed for self injection, so informed consent should be obtained.

Complications of self injection are uncommon. Infection and fibrosis have been reported. ${ }^{8}$ Patients should be told to seek medical advice if their erection lasts more than four hours as there is a risk of priapism. This can be treated by removing blood from the corpus cavernosum with a syringe and injecting an $\alpha$ adrenergic agent such as metaraminol or phenylephrine. Prostaglandin E1 is an alternative to papaverine and may have fewer side effects. ${ }^{9}$ Although self injection is simple and effective and can be offered by a diabetic physician, ${ }^{10}$ some couples find it unacceptable, and long term follow up suggests that many abandon it. ${ }^{112}$

Vacuum tumescence is an effective treatment regardless of the pathogenesis. ${ }^{13} \mathrm{~A}$ rigid cylinder is placed firmly over the penis, and an erection is produced by inducing negative pressure with a hand pump. A constriction band is slipped off the cylinder around the base of the penis to maintain the erection, and the cylinder is removed. Most series suggest that over $70 \%$ of couples can have satisfactory intercourse using this technique. ${ }^{13-15}$ Adverse reactions are uncommon. Some partners have reported that the penis feels cold. Bruising around the penis can occur but is usually self limiting ${ }^{13}$; bleeding diatheses and anticoagulant treatment are therefore contraindications for this treatment. Comparative studies with self injection have suggested that patients find vacuum tumescence as acceptable ${ }^{12}$ or preferable. ${ }^{15}$

Few investigations are required in the assessment of impotent diabetic men. Other treatable causes of impotence such as testicular failure or hyperprolactinaemia should be excluded. More detailed investigation rarely alters management. The problem should be discussed with the patient and his partner. They should be told that impotence in diabetes has a physical cause and will not improve without a physical treatment. Many couples simply require an explanation and decline further treatment.

Those wishing to try a physical treatment should be offered a choice of self injection or vacuum tumescence. Most diabetic men seeking help for impotence are middle aged and have had a stable sexual relationship for many years. Provided both partners are keen to try treatment and have a satisfactory relationship they require only commonsense advice. Most can be treated successfully without specialist psychosexual counselling. ${ }^{101314}$ If a couple is seeking help to try to save a failing relationship treatment is rarely successful and specialist counselling may be required.

A comprehensive diabetes care service should offer to manage impotence as it causes great unhappiness and can be rewarding to treat. As specialist facilities are not required there is no reason why interested general practitioners should not treat impotence. Because many impotent diabetic men are reluctant to ask for help doctors should take the initiative and discreetly inquire about sexual function. Moreover, impotence should have a higher profile in the education of 
diabetic patients to create a greater awareness of available treatments. Once identified, impotent men should be seen, with their partners if possible, in a relaxed sympathetic environment away from the busy diabetic clinic.

D E PRICE Consultant

School of Postgraduate Studies in Medical and Health Care, Morriston Hospital,

Swansea SA6 6NL

1 Price DE, O'Malley BP, James MA, Roshan M, Hearnshaw JR. Why are impotent diabetic men not being treated? Practical Diabetes 1991;8:10-1.

2 British Diabetic Association. What diabetic care to expect. Diabetic Med 1990;7:554.

3 Williams DRR, Home PD. A proposal for continuing audit of diabetes services. Diabetic Med 1991;9:759-64.

4 McCulloch DK, Young RJ, Prescott RJ, Campbell IW, Clarke BF. The natural history of impotence in diabetic men. Diabetologia 1984;26:437-40.
5 Fairburn CG, McCulloch DK, Wu FC. The effects of diabetes on male sexual function. Clinics in Endocrinology and Metabolism 1982;11:749-67.

6 McCulloch DK, Hosking DJ, Tobert A. A pragmatic approach to sexual dysfunction in diabetic men: psychosexual counselling. Diabetic Med 1986;3:485-9.

Wilson SK, Wahman GE, Lange JL. Eleven years' experience with the inflatable penile prosthesis. f Urol 1988;139:951-2.

8 Wiles PG. Erectile impotence in diabetic men: aetiology, investigation and management. Diabetic Med 1992:9:888-92.

9 Diagnostic and therapeutic technology assessment. Vasoactive intracavernous pharmacotherapy for impotence: intracavernous injection of prostaglandin E1. अAMA 1991;265:3321-3.

10 Alexander WD. The diabetes physician and an assessment and treatment programme for male erectile impotence. Diabetic Med 1990;7:540-3.

11 Hollander JB, Gonzalez J, Norman T. Patient satisfaction with pharmacologic erection program Urology 1992;39:439-41.

12 Turner LA, Althof SE, Levine SB, Bodner DR, Kursh ED, Resnick MI. Twelve month comparison of two treatments for erectile dysfunction: self injection versus external vacuum devices. Urology 1992;39:139-44.

13 Price DE, Cooksey G, Jehu D, Bentley S, Hearnshaw JR, Osborn DE. The management of impotence in diabetic men by vacuum tumescence therapy. Diabetic Med 1991;8:964-7.

14 Wiles PG. Successful non-invasive management of erectile impotence in diabetic men. $B M \mathcal{F}$ 1988;296:161-2.

15 Ryder REJ, Robinson A, Anderson J, Parnell L, Ward JD. Intracorporeal papaverine therapy versus vacuum constriction therapy (Erecaid) in the treatment of diabetic impotence [abstract]. Diabetic Med 1992;9(suppl 1):33A.

\title{
Hepatitis B vaccination: protection for how long and against what?
}

\author{
Booster injections are not indicated
}

The purpose of vaccination (used here to mean giving a vaccine) is to prevent disease, but some people would also like to prevent infection. The distinction between infection and disease is not trivial in the case of hepatitis $B$ vaccination. Infection with the virus results in the appearance of core antibody in the serum, and in most people this antibody persists for decades. In addition, those who become carriers of the virus will persistently test positive for surface antigen, and those who become immune will test positive for surface antibody.

Immunisation (used to imply a vaccination that results in a protective immune response) with hepatitis $B$ vaccine results in seropositivity for surface antibody alone. Thus if previously immunised people test positive for core antibody then this indicates infection; if this is associated with persistent surface antigenaemia then the person is at high risk of chronic hepatitis, cirrhosis, and hepatocellular carcinoma. In addition, becoming positive for core antibody may coincide with acute clinical hepatitis.

Immunisation with hepatitis $B$ vaccine is considered to have occurred if concentrations of surface antibody greater than $10 \mathrm{IU} / \mathrm{h}$ develop. Non-response to vaccination is associated with increasing age, male sex, obesity, vaccination in the buttock, and immunosuppression-for example, due to HIV infection and renal dialysis. In these situations repeat doses and increased dosage can result in immunisation. Decay of the putative protective antibody occurs so predictably that when a person's antibody concentration will fall below 10 IU/1 can be predicted from the peak antibody response after immunisation. ${ }^{1}$ For example, a peak antibody concentration of $100 \mathrm{IU} / \mathrm{l}$ can be expected to fall below 10 in about five years, which has been the basis for scheduling booster doses of vaccine.

In this issue Oates et al report a study of hepatitis B vaccination of medical students, which suggests that many students thought that vaccination implied lifelong immunity (p 301). ${ }^{2}$ Were they right or wrong? Is antibody concentration a true marker of protection and does disappearance of antibody equate with becoming susceptible again? More importantly, is this susceptibility to infection or to disease? The answers to these questions lie in long term follow up studies of immunised populations who continue to have a high risk of exposure and in whom surveillance of serological events and acute hepatitis is possible. There are seven such published studies.

Two trials of vaccination to interrupt perinatal transmission showed a vaccine efficacy at 1 year of age of $88 \%$. From these trials Stevens et al followed up for between four and nine years 104 of 235 children who were uninfected at 18 months of age. ${ }^{3}$ None of these children became a carrier of the virus or had a history of acute hepatitis, although $7 \%$ became core antibody positive.

Globally, infection with hepatitis B virus most commonly occurs during childhood. Four studies have examined the duration of protection conferred by vaccination of infants in populations at high risk of childhood infection. In Senegal follow up of children to the age of 7 found that only four out of 135 children were surface antigen positive; whether they were carriers is not known. ${ }^{4}$ In two studies in the Gambia follow up to age 5 has shown frequent infection but no evidence of acute hepatitis. ${ }^{56}$ Although carriers were found in these studies, none were in children with a documented antibody response to the vaccine. In Alaska a study of 1693 children found $100 \%$ protection against carriage of the virus five years after vaccination. ${ }^{7}$ Only four children had evidence of infection, of whom none had associated acute hepatitis.

Two large scale randomised trials in adult homosexual men provide long term information. The first reported on 1100 men at high risk of hepatitis $B$ infection who were randomised to placebo or vaccine derived from plasma. The protective efficacy against infection at 18 months was $92 \%$. Subgroups of those who had been vaccinated in this trial were followed up for longer periods. A subsequent report on 127 of these men who tested negative for HIV-1 showed that none tested positive for hepatitis B surface antigen, although $4 \%$ tested positive for core antibody. ${ }^{8}$ None had suffered clinical acute hepatitis $B$.

In the second trial, the Centers for Disease Control multicentre trial of hepatitis $B$ vaccination, those in the placebo group who remained susceptible at the time of the first analysis were vaccinated. A group of these and those in the vaccinated group are still being followed up. ${ }^{9} A$ total of 733 men were followed up after vaccination was completed; $15 \%$ of those with detectable antibody lost it within this time. Hepatitis B infection occurred in 55 men. In eight this was associated with increased liver enzyme activity and the 